\title{
Are There Signatures of Harmonic Oscillator Shells Far from Stability? First Spectroscopy of ${ }^{110} \mathrm{Zr}$
}

N. Paul, ${ }^{1,2, *}$ A. Corsi,${ }^{1}$ A. Obertelli, ${ }^{1,2}$ P. Doornenbal, ${ }^{2}$ G. Authelet, ${ }^{1}$ H. Baba, ${ }^{2}$ B. Bally, ${ }^{3}$ M. Bender, ${ }^{4}$ D. Calvet, ${ }^{1}$ F. Château, ${ }^{1}$ S. Chen, ${ }^{5,2}$ J.-P. Delaroche, ${ }^{6}$ A. Delbart, ${ }^{1}$ J.-M. Gheller, ${ }^{1}$ A. Giganon, ${ }^{1}$ A. Gillibert, ${ }^{1}$ M. Girod, ${ }^{6}$ P.-H. Heenen, ${ }^{7}$ V. Lapoux, ${ }^{1}$ J. Libert, ${ }^{6}$ T. Motobayashi, ${ }^{2}$ M. Niikura, ${ }^{8}$ T. Otsuka, ${ }^{8}$ T. R. Rodríguez, ${ }^{9}$ J.-Y. Roussé, ${ }^{1}$ H. Sakurai ${ }^{2,8}$ C. Santamaria, ${ }^{1}$ N. Shimizu, ${ }^{10}$ D. Steppenbeck, ${ }^{2}$ R. Taniuchi, ${ }^{2,8}$ T. Togashi, ${ }^{10}$ Y. Tsunoda, ${ }^{10}$ T. Uesaka, ${ }^{2}$ T. Ando, ${ }^{2,8}$ T. Arici, ${ }^{11,12}$ A. Blazhev, ${ }^{13}$ F. Browne, ${ }^{14}$ A. M. Bruce, ${ }^{14}$ R. Carroll, ${ }^{15}$ L. X. Chung, ${ }^{16}$ M. L. Cortés, ${ }^{17,11}$ M. Dewald, ${ }^{13}$ B. Ding, ${ }^{18}$ F. Flavigny, ${ }^{19}$ S. Franchoo, ${ }^{19}$ M. Górska, ${ }^{11}$ A. Gottardo, ${ }^{19}$ A. Jungclaus,${ }^{20}$ J. Lee, ${ }^{21}$ M. Lettmann, ${ }^{17}$ B. D. Linh, ${ }^{16}$ J. Liu, ${ }^{21}$ Z. Liu, ${ }^{18}$ C. Lizarazo, ${ }^{17,11}$ S. Momiyama, ${ }^{2,8}$ K. Moschner ${ }^{13}$ S. Nagamine, ${ }^{8}$ N. Nakatsuka, ${ }^{22}$ C. Nita ${ }^{23}$ C. R. Nobs,${ }^{14}$ L. Olivier, ${ }^{19}$ Z. Patel, ${ }^{15}$ Zs. Podolyák, ${ }^{15}$ M. Rudigier, ${ }^{15}$ T. Saito, ${ }^{8}$ C. Shand ${ }^{15}$ P.-A. Söderström, ${ }^{2}$ I. Stefan, ${ }^{19}$ R. Orlandi, ${ }^{24}$ V. Vaquero, ${ }^{20}$ V. Werner, ${ }^{17}$ K. Wimmer, ${ }^{8}$ and Z. Xu ${ }^{21}$ ${ }^{1} I R F U, C E A$, Université Paris-Saclay, F-91191 Gif-sur-Yvette, France ${ }^{2}$ RIKEN Nishina Center, 2-1 Hirosawa, Wako, Saitama 351-0198, Japan ${ }^{3}$ ESNT, IRFU, CEA, Université Paris-Saclay, F-91191 Gif-sur-Yvette, France

${ }^{4} I P N L$, Université de Lyon, Université Lyon 1, CNRS/IN2P3, F-69622 Villeurbanne, France

${ }^{5}$ School of Physics and State Key Laboratory of Nuclear Physics and Technology, Peking University, Beijing 100871, People's Republic of China ${ }^{6}$ CEA, DAM, DIF, F-91297 Arpajon, France

${ }^{7}$ PNTPM, CP229, Université Libre de Bruxelles, B-1050 Bruxelles, Belgium ${ }^{8}$ Department of Physics, University of Tokyo, 7-3-1 Hongo, Bunkyo, Tokyo 113-0033, Japan

${ }^{9}$ Departamento de Física Teorica, Universidad Autónoma de Madrid, 28049 Madrid, Spain

${ }^{10}$ Center for Nuclear Study, University of Tokyo, 7-3-1 Hongo, Bunkyo, Tokyo 113-0033, Japan

${ }^{11}$ GSI Helmholtzzentrum für Schwerionenforschung GmbH, 64291 Darmstadt, Germany

${ }^{12}$ Justus-Liebig-Universität Giessen, D-35392 Giessen, Germany

${ }^{13}$ Institut für Kernphysik, Universität zu Köln, 50937 Köln, Germany

${ }^{14}$ School of Computing Engineering and Mathematics, University of Brighton, Brighton BN2 4GJ, United Kingdom

${ }^{15}$ Department of Physics, University of Surrey, Guildford GU2 7XH, United Kingdom

${ }^{16}$ Institute for Nuclear Science and Technology, VAEI, 179 Hoang Quoc Viet, Cau Giay, Hanoi, Vietnam

${ }^{17}$ Institut für Kernphysik, Technische Universität Darmstadt, 64289 Darmstadt, Germany

${ }^{18}$ Institute of Modern Physics, Chinese Academy of Sciences, Lanzhou 730000, People's Republic of China

${ }^{19}$ Institut de Physique Nucléaire, CNRS-IN2P3, Université Paris-Sud, Université Paris-Saclay, 91406 Orsay Cedex, France

${ }^{20}$ Instituto de Estructura de la Materia, CSIC, 28006 Madrid, Spain

${ }^{21}$ Department of Physics, The University of Hong Kong, Pokfulam, Hong Kong

${ }^{22}$ Department of Physics, Faculty of Science, Kyoto University, Kyoto 606-8502, Japan

${ }^{23}$ Horia Hulubei National Institute of Physics and Nuclear Engineering (IFIN-HH), RO-077125 Bucharest, Romania

${ }^{24}$ Advanced Science Research Center, Japan Atomic Energy Agency, Tokai, Ibaraki, 319-1195, Japan

(Received 16 November 2016; published 18 January 2017)

The first measurement of the low-lying states of the neutron-rich ${ }^{110} \mathrm{Zr}$ and ${ }^{112} \mathrm{Mo}$ was performed via inbeam $\gamma$-ray spectroscopy after one proton removal on hydrogen at $\sim 200 \mathrm{MeV} /$ nucleon. The $2_{1}^{+}$excitation energies were found at $185(11) \mathrm{keV}$ in ${ }^{110} \mathrm{Zr}$, and $235(7) \mathrm{keV}$ in ${ }^{112} \mathrm{Mo}$, while the $R_{42}=E\left(4_{1}^{+}\right) / E\left(2_{1}^{+}\right)$ ratios are 3.1(2), close to the rigid rotor value, and 2.7(1), respectively. These results are compared to modern energy density functional based configuration mixing models using Gogny and Skyrme effective interactions. We conclude that first levels of ${ }^{110} \mathrm{Zr}$ exhibit a rotational behavior, in agreement with previous observations of lighter zirconium isotopes as well as with the most advanced Monte Carlo shell model predictions. The data, therefore, do not support a harmonic oscillator shell stabilization scenario at $Z=40$ and $N=70$. The present data also invalidate predictions for a tetrahedral ground state symmetry in ${ }^{110} \mathrm{Zr}$.

DOI: 10.1103/PhysRevLett.118.032501

Nuclei, like atoms, manifest quantized energy states that can be interpreted in terms of an underlying shell structure-a convenient but non-observable theoretical construct $[1,2]$. Within the classical picture, large gaps between adjacent shells give rise to particularly stable configurations whose proton and neutron numbers are traditionally called "magic" [3]. The magic numbers for stable nuclei were first successfully described by invoking a one-body square-well 
and spin-orbit potential [4,5]; the latter was eventually replaced by a harmonic oscillator potential with $l^{2}$ term to obtain proper angular momentum splittings [6]. However, studies of radioactive nuclei over the past decades have shown that the magic numbers are not universal across the nuclear chart [7-10]. Despite intensive effort, the theoretical description of these structural changes is not yet fully understood and the mechanisms that drive structural evolution differ between models. Within the shell-model picture, the tensor and central forces modify single-particle energies via interactions between valence proton and neutron orbitals according to their filling and relative spin-to-orbital orientation $[3,11-18]$. In a meanfield conception, spherical shell gaps may be modified far from stability by increased surface diffuseness. Its principal effect is a quenching of the spin-orbit splittings by as much as $40 \%$ near the dripline [19,20], but other features of shell structure are affected as well, such that shell effects might even be effaced entirely when approaching the neutron dripline [21]. Tensor interactions add additional local variations to the spin-orbit splittings [22]. In the mean-field picture, the competition between spherical and deformed shapes is illustrated by the appearance of deformed gaps in the single-particle spectrum as a function of intrinsic deformation. Large gaps may lead to shell stabilization. Qualitatively, if the spin-orbit splitting that gives rise to the $N=82$ shell gap is reduced by these mechanisms far from stability, the harmonic oscillator gap at $N=70$ may open up instead [23]. If this happens already for modest neutron-to-proton ratios, then it may be manifest at ${ }^{110} \mathrm{Zr}$, whose 40 protons and 70 neutrons combine two harmonic oscillator shell closures [24]. This makes ${ }^{110} \mathrm{Zr}$ a prime benchmark for the dynamic interplay between shell structure and multipole correlations far from stability.

A shell-stabilized ${ }^{110} \mathrm{Zr}$ has potential implications for our understanding of the rapid neutron capture process. Despite recent improvements from new $\beta$-decay lifetimes [25], $r$-process calculations consistently fail to reproduce the elemental abundance distribution near mass 110 . Currently this discrepancy is an entanglement of astrophysical and nuclear structure predictions, but a shellstabilized ${ }^{110} \mathrm{Zr}$ is one proposed solution to this anomaly [24]. Spectroscopic information near ${ }^{110} \mathrm{Zr}$ will help delineate the $N=70,82$ shell evolution and constrain the structure models used in $r$-process simulations.

On both the theoretical and experimental sides, ${ }^{110} \mathrm{Zr}$ has motivated numerous studies while its structure remains unknown. A shell-stabilized ${ }^{110} \mathrm{Zr}$ has been predicted by independent mean-field and microscopic-macroscopic approaches [26-28], which interestingly all find that the shell-stabilization coincides with a tetrahedral configuration. This exotic symmetry, hitherto unobserved, is expected to compete strongly with deformed minima and its emergence is known to be very sensitive to pairing effects [29]. If a tetrahedral configuration persists in the ground state of ${ }^{110} \mathrm{Zr}$; however, it would manifest a unique energy spectrum [30,31], distinguishable in a first-spectroscopy measurement. Meanwhile, most predictions of the ${ }^{110} \mathrm{Zr}$ ground state show well-deformed prolate $[32,33]$ or shape coexistent minima [34-39], though the exact structure is highly sensitive to the details of the effective interaction [22]. Recently, a strong deformation of ${ }^{110} \mathrm{Zr}$, similar to lighter $\mathrm{Zr}$ isotopes beyond $N=60$, has been predicted by Monte Carlo shell model (MCSM) calculations [40].

Experimentally, the weakening of the $N=82$ shell closure was first claimed from $\beta-\gamma$ decay spectroscopy of ${ }^{130} \mathrm{Cd}$ and ${ }^{130} \mathrm{In}$ where the measured $Q_{\beta}$ value agreed best with predictions from a shell-quenched mass model [41]. This conclusion was challenged by measurements of isomeric decays in ${ }^{130} \mathrm{Cd}$ which showed no evidence of shell quenching [42]. More recently, mass measurements of ${ }^{129-131} \mathrm{Cd}$ show a reduction of neutron separation energy differences by $1 \mathrm{MeV}$ going from ${ }^{132} \mathrm{Sn}$ to ${ }^{130} \mathrm{Cd}$ [43]. Thus far experiments near ${ }^{110} \mathrm{Zr}$, including the $\beta$-decay half-lives of ${ }^{106-112} \mathrm{Zr}$ [25], lifetime measurements of the $2_{1}^{+}$states in ${ }^{104,106} \mathrm{Zr}$ [44], and spectroscopy of the low-lying excited states of ${ }^{108} \mathrm{Zr}$ [45], show no hint of a shell gap at $N=70$ and suggest that the $\mathrm{Zr}$ isotopes with $N>60$ are prolate deformed. In this Letter we present the first spectroscopic evidence of the deformed nature of ${ }^{110} \mathrm{Zr}$ and ${ }^{112} \mathrm{Mo}$, and discuss the origin of their collectivity through state-of-theart microscopic models.

The measurement was performed at the Radioactive Isotope Beam Factory operated by the RIKEN Nishina Center for Accelerator-Based Science and the Center for Nuclear Study of the University of Tokyo. A $30 \mathrm{pnA}{ }^{238} \mathrm{U}$ primary beam was accelerated to $345 \mathrm{MeV} /$ nucleon. The radioactive isotope beams were created via in-flight fission of the primary beam on a $3-\mathrm{mm}$ thick ${ }^{9} \mathrm{Be}$ target positioned at the focal plane before the BigRIPS two-stage fragment separator [46]. The isotopes of interest were selected from the secondary cocktail beam using the $B \rho-\Delta E-B \rho$ method [47]. These isotopes, magnetically centered on ${ }^{111} \mathrm{Nb}$, impinged on a 99(1) mm-thick liquid hydrogen $\left(\mathrm{LH}_{2}\right)$ target with an incident kinetic energy of $260 \mathrm{MeV} /$ nucleon. The energy loss in the hydrogen target was approximately $100 \mathrm{MeV} /$ nucleon. Individual intensities for the secondary beams of interest ${ }^{113} \mathrm{Tc}$ and ${ }^{111} \mathrm{Nb}$ were 32 and 20 particles per second, respectively. Nuclei before and after the $\mathrm{LH}_{2}$ target were unambiguously identified in the BigRIPS and ZeroDegree spectrometers, respectively, using the TOF $-B \rho-\Delta E$ method [47]. ${ }^{110} \mathrm{Zr}$ and ${ }^{112} \mathrm{Mo}$ were created via proton removal in the $\mathrm{LH}_{2}$ target. Emitted $\gamma$ rays were detected with the DALI2 gamma array, which consisted of $186 \mathrm{NaI}(\mathrm{Tl})$ detectors covering polar angles of $12^{\circ}-118^{\circ}$ with an average angular resolution of $6^{\circ}$ [48]. The full-energy peak detection 
efficiency with no addback was simulated to be $31 \%$ for $500 \mathrm{keV} \gamma$ rays emitted in flight [49]. DALI2 was calibrated with ${ }^{152} \mathrm{Eu},{ }^{60} \mathrm{Co},{ }^{137} \mathrm{Cs},{ }^{88} \mathrm{Y}$, and ${ }^{133} \mathrm{Ba}$ sources, yielding calibration peaks from 121 to $1332 \mathrm{keV}$, a calibration error of $1.5 \mathrm{keV}$, and energy resolution of $59 \mathrm{keV}$ full width at half maximum (FWHM) for the $662 \mathrm{keV}$ peak of ${ }^{137} \mathrm{Cs}$, consistent with Ref. [50]. DALI2 thresholds were set at $100 \mathrm{keV}$ on average. A $300-\mathrm{mm}$ long time projection chamber was placed around the $\mathrm{LH}_{2}$ target in a setup known as MINOS [51]. MINOS detected knocked-out protons with an efficiency to detect at least one of the two protons from $(p, 2 p)$ simulated at $95 \%$. The proton tracks were used to reconstruct the reaction vertex with a precision of $5 \mathrm{~mm}$ FWHM. Knowledge of the vertex allowed precise Doppler correction of the $\gamma$ rays detected in DALI2, as demonstrated in Ref. [52].

The Doppler-corrected $\gamma$-ray spectra for ${ }^{112} \mathrm{Mo}$ and ${ }^{110} \mathrm{Zr}$ are shown in Fig. 1. The transitions of interest lie close in energy to the bremsstrahlung spectrum generated from fast beam particles colliding with hydrogen atoms in the target. This component was measured in coincidence with unreacted beam particles, normalized according to the number of nuclei incident on the $\mathrm{LH}_{2}$ target, and subtracted from the experimental spectra to clearly identify the peaks of interest. The peaks that emerge after subtraction are visible at forward angles without subtraction. The subtraction method was validated on ${ }^{86} \mathrm{Ge}$ from the same

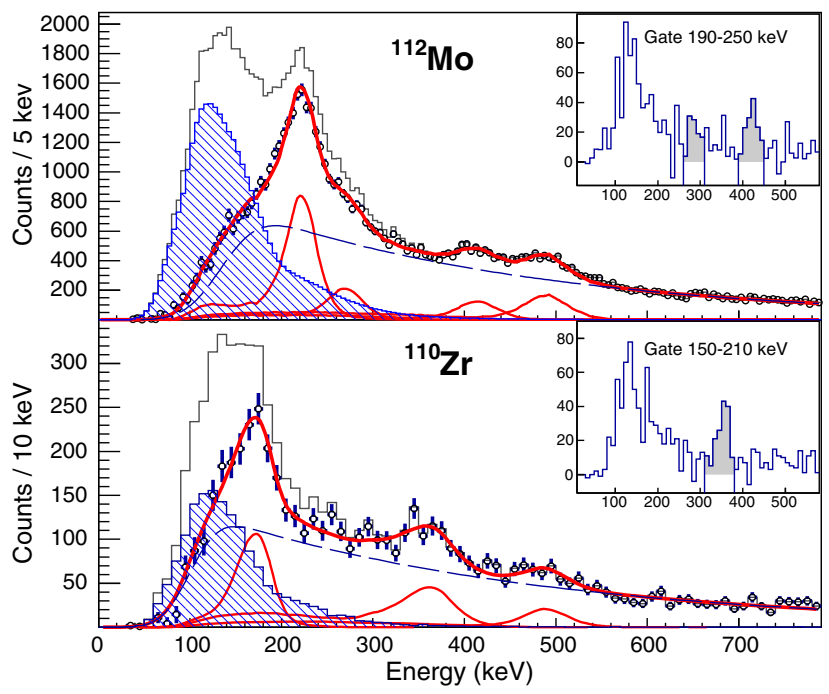

FIG. 1. Doppler-corrected $\gamma$-ray spectra of ${ }^{112} \mathrm{Mo}$ and ${ }^{110} \mathrm{Zr}$ including the total spectrum (solid black line), normalized bremsstrahlung component (blue), and the subtracted spectrum (open circles). The fit to the subtracted spectrum is shown by the thick red line, individual simulated responses are shown by thin red lines, while the exponential background is shown by the dashed line. (Insets) Background subtracted $\gamma-\gamma$ coincidences for the $2_{1}^{+} \rightarrow 0_{1}^{+}$transitions. The inset for ${ }^{112} \mathrm{Mo}$ is restricted to $\gamma$ multiplicities lower than 4 for a better signal-to-background ratio. Coincident peaks are highlighted in gray. experiment, where the $2_{1}^{+} \rightarrow 0_{1}^{+}$transition is known to be well separated from the bremsstrahlung spectrum at $527 \mathrm{keV}$ [53], and it was verified that the method did not create any artificial peaks. The subtracted spectra were then fit with response functions simulated using GEANT4 [49]. The simulation of the DALI2 detector response functions was optimized for accuracy in the low-energy region of interest by including individual DALI2 thresholds and accounting for absorption of the $\gamma$ rays in all materials surrounding the target. The remaining background, originating from unresolved high energy transitions and particle induced background, has an unknown shape at low energy. It was taken to be an exponential cutoff with an error function, corresponding to the effect of the DALI2 thresholds. The parameters of the exponential background were fit simultaneously with the response function intensities.

Considering the case of ${ }^{112} \mathrm{Mo}$, three major peaks were visible in the data, and a fourth was inferred from the shoulder on the high-energy side of the strongest transition. A matrix of response functions was simulated for each peak, corresponding to the possible combinations of transition energies and level lifetimes. The subtracted spectrum was then fit with all combinations of the four peak arrays. The Pearson's $\chi^{2}$ from the fitting procedure was converted to probability assuming a multivariate Gaussian probability density function, and the most probable response function and corresponding one- $\sigma$ regions of confidence in the energy-lifetime plane were extracted for each peak. The found transition energies are 235(7), $410(11)$, and 485(26) $\mathrm{keV}$ for the three major peaks. The uncertainties include the statistical region of confidence from the fitting procedure, intrinsic energy resolution, and systematic uncertainties added in quadrature. Note that the ambiguity induced by the unknown shape of the lowenergy background does not allow us to determine lifetimes from the peak shapes of the measured transitions with significant accuracy. An identical procedure was followed for ${ }^{110} \mathrm{Zr}$ for the three peaks visible in the data, found at $185(11), 380(21)$, and $485(11) \mathrm{keV}$. In addition, ${ }^{108} \mathrm{Zr}$ (not shown) was analyzed as a reference measurement. The $2_{1}^{+}$excitation energy in ${ }^{108} \mathrm{Zr}$ is found at $170(11) \mathrm{keV}$, consistent with Ref. [45], thus providing a validation of the present analysis.

The $\gamma-\gamma$ spectra allow us to construct the level schemes shown in Fig. 2. The $\gamma-\gamma$ coincidences gated on the $2_{1}^{+} \rightarrow 0_{1}^{+}$transitions are shown in the insets of Fig. 1 . For both ${ }^{110} \mathrm{Zr}$ and ${ }^{112} \mathrm{Mo}$, the transition near $500 \mathrm{keV}$ is not in coincidence with the other two major peaks and its intensity evolution as a function of multiplicity is consistent with a transition directly to the ground state. Therefore, it is taken as the $2_{2}^{+} \rightarrow 0_{1}^{+}$transition. The $2_{2}^{+} \rightarrow 2_{1}^{+}$transition in ${ }^{112}$ Mo corresponds with the shoulder visible to the right of the $2_{1}^{+} \rightarrow 0_{1}^{+}$peak, and is visible in the $\gamma-\gamma$ coincidences as the highlighted peak near $300 \mathrm{keV}$; however, no clear 

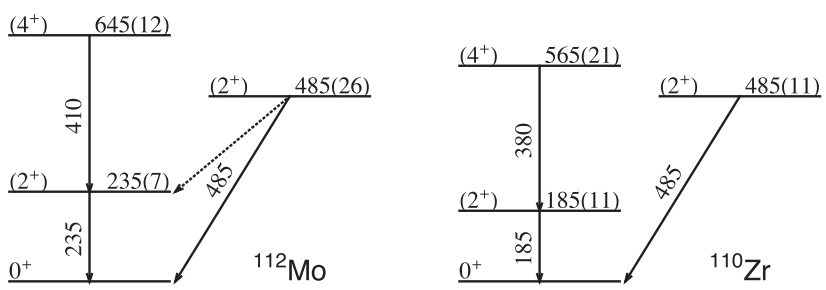

FIG. 2. Level schemes for ${ }^{112} \mathrm{Mo}$ and ${ }^{110} \mathrm{Zr}$ established in this work. Energies are given in $\mathrm{keV}$.

minimum in the $\chi^{2}$ surface was found from the fitting procedure. The energy of the response function shown in the fit in Fig. 1 is $280 \mathrm{keV}$. With the available statistics, we do not observe the $2_{2}^{+} \rightarrow 2_{1}^{+}$transition in ${ }^{110} \mathrm{Zr}$. For both ${ }^{112} \mathrm{Mo}$ and ${ }^{110} \mathrm{Zr}$, the nonobservation of the $2_{2}^{+} \rightarrow 0_{2}^{+}$ transition suggests that the $0_{2}^{+}$state lies higher, or very close in energy to the $2_{2}^{+}$.

The experimental values of the $2_{1}^{+}$excitation energies are shown in Fig. 3 for $N=70$ isotones from $\mathrm{Zr}$ to $\mathrm{Sm}$ $(Z=62)$. The $2_{1}^{+}$energy peaks at closed shell ${ }^{120} \mathrm{Sn}$ and decreases symmetrically for both larger and smaller proton numbers towards maximum collectivity at midshell. Towards the neutron dripline, the $2_{1}^{+}$energies of ${ }^{112} \mathrm{Mo}$ and ${ }^{110} \mathrm{Zr}$ measured in this work show a steep decrease.

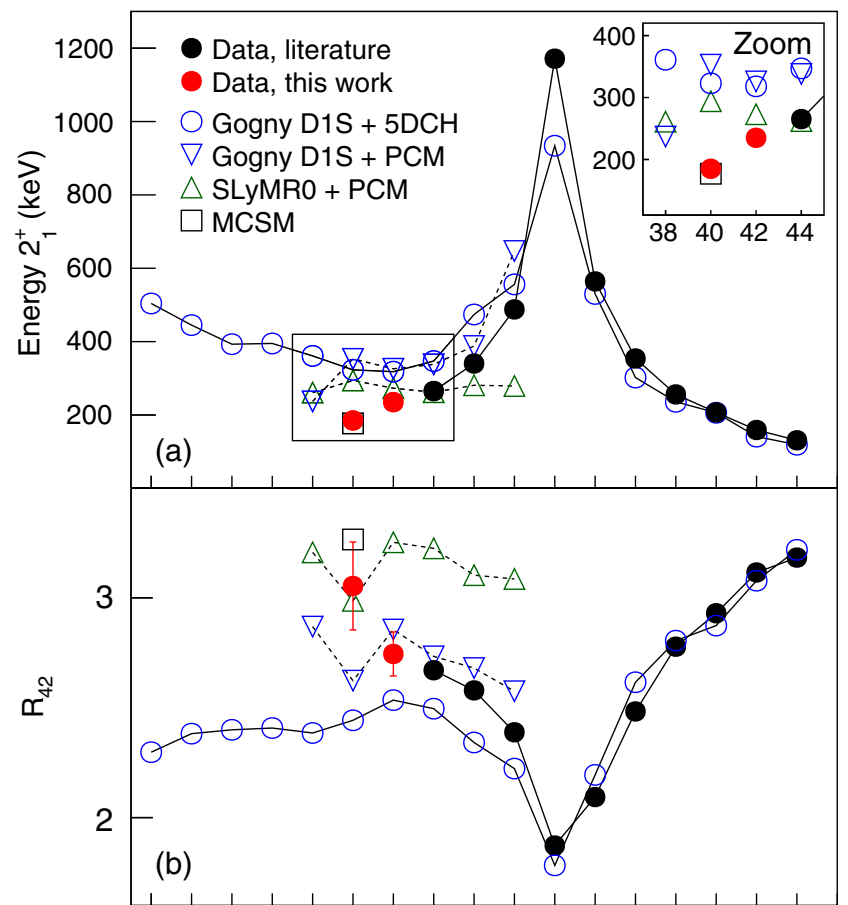

303234363840424446485052545658606264 Z

FIG. 3. Experimental $2_{1}^{+}$energies (a) and $R_{42}$ ratios (b) for the $N=70$ isotones compared with theory: 5DCH [35] and PCM [54] with Gogny D1S interaction, PCM with Skyrme SLyMR0 [55], and MCSM calculations [40]. Experimental data are taken from Ref. [53] and this work.
Similarly, the experimental $R_{42}=E\left(4_{1}^{+}\right) / E\left(2_{1}^{+}\right)$ratios shown in the lower panel of Fig. 3 suggest increased collectivity towards ${ }^{110} \mathrm{Zr}$ with a value of $3.1(2)$. This pattern is consistent with a transition from a harmonic vibrator to a deformed symmetric rotor towards midshell as one departs from the $Z=50$ shell closure. Assuming that the next proton shell closure below ${ }^{120} \mathrm{Sn}(Z=50)$ occurs in ${ }^{98} \mathrm{Ni}(Z=28)$, expected to be beyond the neutron dripline [56], the maximum of collectivity would take place at $Z \sim 39$ close to ${ }^{110} \mathrm{Zr}$, consistent with our results. The present measurement shows no sign of a subshell closure at $Z=40$ along the $N=70$ isotonic chain.

Likewise, the systematics of $2_{1}^{+}$energies along the $\mathrm{Zr}$ chain are smooth as a function of neutron number with no particular increase of $2_{1}^{+}$value from ${ }^{108} \mathrm{Zr}$ [45] to ${ }^{110} \mathrm{Zr}$, showing no sign of any shell effect at $N=70$ either, and demonstrating that ${ }^{110} \mathrm{Zr}$ is a well-deformed nucleus with no significant structural change from lighter ${ }^{100-108} \mathrm{Zr}$ isotopes.

In order to further investigate the structure of ${ }^{112} \mathrm{Mo}$ and ${ }^{110} \mathrm{Zr}$, we compared our results to state-of-the-art beyondmean-field calculations [57]. The 5-Dimension Collective Hamiltonian $(5 \mathrm{DCH})$ with the Gogny D1S effective interaction $[62,63]$ reproduces well collective properties across the nuclear landscape [35]. Experimental systematics of $2_{1}^{+}$ energies as well as $R_{42}$ ratios are compared to predictions in Fig. 3. Rather constant underprediction of collectivity is found between $Z=48$ and 42 , but ${ }^{112} \mathrm{Mo}$ and ${ }^{110} \mathrm{Zr}$, in particular, mark a clear departure from the theoretical trend showing significantly lower $2_{1}^{+}$energies and higher $R_{42}$ ratios than predictions. In ${ }^{120} \mathrm{Sn}$, the Gogny D1S interaction gives a spherical gap between the $2 p_{1 / 2}$ and $1 g_{9 / 2}$ orbitals $(Z=40)$ of $3.2 \mathrm{MeV}$, and a gap between the $3 s_{1 / 2}$ and $1 h_{11 / 2}$ orbitals $(N=70)$ of $1.0 \mathrm{MeV}$. The same gaps are 3.0 and $2.1 \mathrm{MeV}$, respectively, for ${ }^{110} \mathrm{Zr}$, showing a sizable neutron gap away from stability, yet a deformed minimum is energetically preferred both at the mean-field and beyond-mean-field levels.

We confirm that the $2_{1}^{+}$and $R_{42}$ disagreement between theory and experiment for the two nuclei studied does not stem from the $5 \mathrm{DCH}$ approximations by comparing with predictions from projected configuration mixing (referred to as PCM in the following [also called symmetryconserving configuration mixing model (SCCM) [54] or projected generator coordinate method (pGCM) [64].]) using the same effective interaction. PCM takes into account triaxial degrees of freedom, as does the $5 \mathrm{DCH}$ approach, but solves exactly the Griffin-Hill-Wheeler equation in the deformation coordinates for particlenumber and angular-momentum projected Bogoliubov states. Excitation energy predictions from the two models are shown in Fig. 4 and are very similar. However, D1S + PCM calculations predict a lowering of the $2_{1}^{+}$energy for ${ }^{108} \mathrm{Sr}$, in better agreement with the trend observed in the 


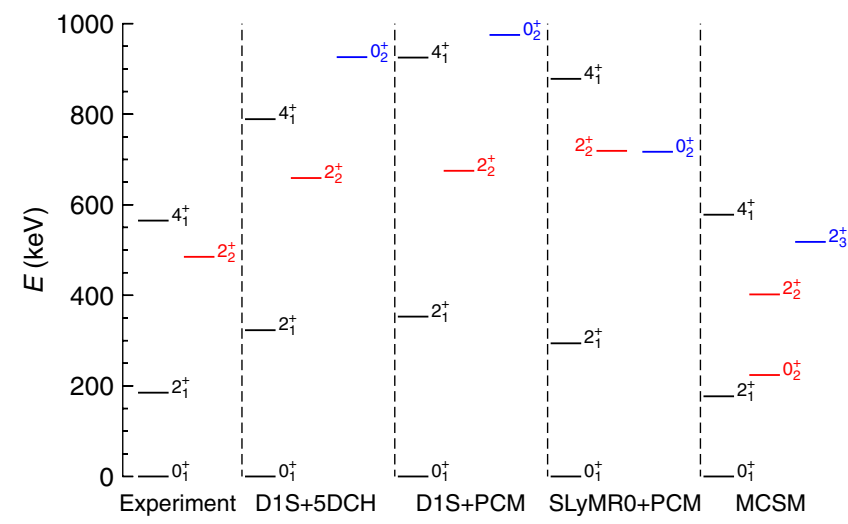

FIG. 4. Comparison between experimental and theoretical low-lying states in ${ }^{110} \mathrm{Zr}$ from this work.

data. D1S + PCM calculations also better reproduce the $R_{42}$ trends, but both calculations clearly underpredict the collectivity of ${ }^{110} \mathrm{Zr}$. The dependence on the effective interaction is illustrated by PCM calculations using the SLyMR0 force, a recent Skyrme parametrization [55,65]. The systematics of $2_{1}^{+}$energies are rather flat from $40 \leq Z \leq 50$, missing the trend towards ${ }^{120} \mathrm{Sn}$ while approaching but still overestimating our measured the $2_{1}^{+}$ energies for ${ }^{110} \mathrm{Zr}$ and ${ }^{112} \mathrm{Mo}$.

For the case of ${ }^{110} \mathrm{Zr}$, a detailed comparison between our data and the theoretical level schemes is shown in Fig. 4. All the above calculations predict triaxial ground and $2_{1,2}^{+}$ states built on rather $\gamma$-soft potential energy surfaces [57]. The $2_{2}^{+}$state is predicted below the $4_{1}^{+}$, in qualitative agreement with the data, though the excitation energies are globally overpredicted, a trend already illustrated in Fig. 3.

Our data also suggest that the ground state band we observe is not of a tetrahedral character. According to calculations by Tagami et al. [30,31], the lowest-lying levels of the tetrahedral band in ${ }^{110} \mathrm{Zr}$ are predicted to be $\left(3^{-}, 4^{+}, 6^{+}\right)$. However, the low energy of the transitions we measure, combined with the fact that we observe the $\gamma$ decay in flight, excludes the possibility that our most intense gamma ray results from $E 3$ and higher multipolarity transitions. The only candidates then for the $\gamma$ rays we observe would be the $4^{+}$to $3^{-}$and $6^{+}$to $4^{+}$transitions, but these are predicted to have a $R_{42}$ ratio of $\sim 2$, very far from our measured ratio of $\sim 3$. Furthermore, the spectral systematics along the $\mathrm{Zr}$ isotopic chain allow a direct mapping of our strongest transitions to the $2^{+}$and $4^{+}$levels in ${ }^{108} \mathrm{Zr}$, which exhibits a nearly identical ground state band [45]. For these reasons, we reject the interpretation of the ground state band as being built upon a tetrahedraldeformed minimum as suggested by Ref. [27]; however, we cannot exclude the existence of an excited tetrahedral band, as in Ref. [30].

To further quantify the influence of the effective interaction on the above observations, we performed a local sensitivity test within the $5 \mathrm{DCH}$ approach by increasing the spin-orbit term of the Gogny D1S interaction from -130 to $-140 \mathrm{MeV} \mathrm{fm}^{5}$, with no change of the other terms of the interaction. Within this model, modification of the spin-orbit strength is an efficient, but nonunique, way to artificially increase the deformation and see the spectroscopic effects. The results show a lowering of the $2_{1}^{+}$ excitation energies down to 259 and $215 \mathrm{keV}$ for ${ }^{112} \mathrm{Mo}$ and ${ }^{110} \mathrm{Zr}$, respectively, in much better agreement with experimental values. The agreement for $R_{42}$ is also improved for these two isotones, going from 2.4 to 2.8 for ${ }^{110} \mathrm{Zr}$, approaching the experimental value of 3 . For the case of ${ }^{110} \mathrm{Zr}$, the collective wave function of the $2_{1}^{+}$state shifts from a triaxial maximum at $\beta=0.25$ to prolate at $\beta=0.4$, concurrently increasing the $g_{9 / 2}$ occupancy by 0.3 protons at the prolate minimum of the potential energy surface, consistent with increased collectivity.

Our results are finally compared to MCSM predictions for ${ }^{110} \mathrm{Zr}$ [40], also shown in Figs. 3 and 4. Predictions of excitation energies for the $2_{1}^{+}, 4_{1}^{+}$states of ${ }^{110} \mathrm{Zr}$ are in good agreement with our measured values. MCSM operates within the paradigm of the so-called type $I I$ shell evolution [66], wherein the low-lying spectroscopy of $\mathrm{Zr}$ isotopes with $N \geq 60$ is understood as coming from a modification of the neutron single particle energies driven by the deformationtriggered promotion of protons from the $f p$ shell to the $g_{9 / 2}$ orbital. This is consistent with the results of the sensitivity study, where improved agreement is obtained for a larger deformation, when more protons are promoted into the $g_{9 / 2}$ orbital. However, the MCSM calculations for ${ }^{110} \mathrm{Zr}$ predict the $0_{2}^{+}$state below the $2_{2}^{+}$state, at variance with the energy density functional based models. The identification of the $0_{2}^{+}$ state, of particular interest for a fine understanding of the structure of ${ }^{110} \mathrm{Zr}$, requires further experiments.

In conclusion, we performed the first spectroscopy of the neutron-rich $N=70$ isotones ${ }^{112} \mathrm{Mo}$ and ${ }^{110} \mathrm{Zr}$. Low $2_{1}^{+}$ excitation energies found at 235(7) and 185(11) keV, respectively, as well as $R_{42}$ values of $\sim 3$, clearly indicate that both of these nuclei are well deformed. The present study demonstrates that ${ }^{110} \mathrm{Zr}$ experiences no stabilizing shell effect corresponding to the harmonic oscillator nucleon magic numbers $Z=40$ and $N=70$, and seems to rule out this proposed solution to the $r$-process anomaly at $A=110$. While energy density functional based predictions using the Gogny D1S interaction along the $N=70$ isotonic chain show an overall good agreement with the $2_{1}^{+}, 4_{1}^{+}$systematics for $Z \geq 42$, a clear underprediction of collectivity is evidenced for ${ }^{110} \mathrm{Zr}$. The roots of this discord remain to be understood. Eventually, the spectroscopy of ${ }^{60} \mathrm{Ca}$ with $Z=$ 20 and $N=40$ should further our understanding of harmonic oscillator shell effects at the neutron dripline [67-69].

We express our gratitude to the RIKEN Nishina Center accelerator staff for providing the stable and high-intensity 
uranium beam and to the BigRIPS team for the smooth operation of the secondary beams. N. P. thanks G. Schnabel for help with the statistics component of this work. A. O. thanks the European Research Council for its support of the MINOS project through ERC Grant No. MINOS-258567. PCM calculations using SLyMR0 were performed using HPC resources from GENCI-TGCC (Grants No. 2016057392 and No. 2016-050707) and CCRT supercomputer Airain. MCSM calculations were supported in part by JSPS Grants-in-Aid for Scientific Research (23244049), in part by HPCI Strategic Program (hp150224), in part by MEXT and JICFuS and Priority Issue on Post-K computer (Elucidation of the fundamental laws and evolution of the universe) (hp160211), and by the CNS-RIKEN joint project for large-scale nuclear structure calculations. T. R. R. acknowledges support from GSI-Darmstadt computing facilities and the financial support of Ministerio de Economa y Competitividad under Contract No. FIS2014-53434 and Programa Ramon y Cajal 2012 No. 11420. S. C. acknowledges support of the IPA program at RIKEN Nishina Center. K. M. acknowledges support from the German BMBF Grant No. 05P15PKFNA. M. L. C., M. L., and V. W. acknowledge support from the German BMBF Grant No. 05P12RDFN8. L. X. C. and B. D. L. acknowledge support from the Vietnam Ministry of Science and Technology, and the Radioactive Isotope Physics Laboratory of the RIKEN Nishina Center for supporting their stay during the experiment. A. J. and V. V. acknowledge support from the Spanish Ministerio de Economa y Competitividad under Contract No. FPA2014-57196-C5-4P. U.K. participants acknowledge support from the Science and Technology Facilities Council (STFC).

*nancy.paul@cea.fr

[1] R. Furnstahl and H.-W. Hammer, Phys. Lett. B 531, 203 (2002).

[2] T. Duguet, H. Hergert, J. D. Holt, and V. Somà, Phys. Rev. C 92, 034313 (2015).

[3] O. Sorlin and M.-G. Porquet, Prog. Part. Nucl. Phys. 61, 602 (2008).

[4] M. G. Mayer, Phys. Rev. 75, 1969 (1949).

[5] O. Haxel, J. H. D. Jensen, and H. E. Suess, Phys. Rev. 75, 1766 (1949).

[6] S. G. Nilsson, Dan. Mat. Fys. Medd. 16, 1 (1955).

[7] C. Détraz, D. Guillemaud, G. Huber, R. Klapisch, M. Langevin, F. Naulin, C. Thibault, L. C. Carraz, and F. Touchard, Phys. Rev. C 19, 164 (1979).

[8] B. Bastin, S. Grévy, D. Sohler, O. Sorlin, Z. Dombrádi et al., Phys. Rev. Lett. 99, 022503 (2007).

[9] A. Obertelli et al., Phys. Lett. B 633, 33 (2006).

[10] D. Steppenbeck et al., Nature (London) 502, 207 (2013).

[11] P. Federman and S. Pittel, Phys. Lett. B 69, 385 (1977).

[12] P. Federman, S. Pittel, and R. Campos, Phys. Lett. B 82, 9 (1979).
[13] T. Otsuka, T. Matsuo, and D. Abe, Phys. Rev. Lett. 97, 162501 (2006).

[14] T. Otsuka, R. Fujimoto, Y. Utsuno, B. A. Brown, M. Honma, and T. Mizusaki, Phys. Rev. Lett. 87, 082502 (2001).

[15] T. Otsuka, T. Suzuki, R. Fujimoto, H. Grawe, and Y. Akaishi, Phys. Rev. Lett. 95, 232502 (2005).

[16] T. Otsuka, T. Suzuki, M. Honma, Y. Utsuno, N. Tsunoda, K. Tsukiyama, and M. Hjorth-Jensen, Phys. Rev. Lett. 104, 012501 (2010).

[17] Y. Tsunoda et al., Phys. Rev. C 89, 031301(R) (2014).

[18] N. A. Smirnova, B. Bally, K. Heyde, F. Nowacki, and K. Sieja, Phys. Lett. B 686, 109 (2010).

[19] G. A. Lalazissis, D. Vretenar, W. Pöschl, and P. Ring, Phys. Lett. B 418, 7 (1998).

[20] J. Dobaczewski, I. Hamamoto, W. Nazarewicz, and J. A. Sheikh, Phys. Rev. Lett. 72, 981 (1994).

[21] W. Nazarewicz and R. Casten, Nucl. Phys. A682, 295 (2001).

[22] M. Bender, K. Bennaceur, T. Duguet, P. H. Heenen, T. Lesinski, and J. Meyer, Phys. Rev. C 80, 064302 (2009).

[23] B. Pfeiffer, K.-L. Kratz, and J. Dobaczewski, Acta Phys. Pol. B 27, 475 (1996).

[24] K. L. Kratz, B. Pfeiffer, O. Arndt, S. Hennrich, and A. Wöhr, Eur. Phys. J. A 25, 633 (2005).

[25] G. Lorusso et al., Phys. Rev. Lett. 114, 192501 (2015).

[26] J. Dudek, A. Gozdz, N. Schunck, and M. Miskiewicz, Phys. Rev. Lett. 88, 252502 (2002).

[27] N. Schunck, J. Dudek, A. Góźdź, and P. H. Regan, Phys. Rev. C 69, 061305 (2004).

[28] J. Zhao et al., arXiv:1606.08994v1 [Phys. Rev. C (to be published)].

[29] K. Zberecki, P.-H. Heenen, and P. Magierski, Phys. Rev. C 79, 014319 (2009).

[30] S. Tagami, Y. R. Shimizu, and J. Dudek, Phys. Rev. C 87, 054306 (2013).

[31] S. Tagami, M. Shimada, Y. Fujioka, Y. R. Shimizu, and J. Dudek, Phys. Scr. 89, 054013 (2014).

[32] L. Geng, H. Toki, S. Sugimoto, and J. Meng, Prog. Theor. Phys. 110, 921 (2003).

[33] B. Sorgunlu and P. Van Isacker, Nucl. Phys. A808, 27 (2008).

[34] M. Kortelainen, T. Lesinski, J. Moré, W. Nazarewicz, J. Sarich, N. Schunck, M. V. Stoitsov, and S. Wild, Phys. Rev. C 82, 024313 (2010).

[35] J. P. Delaroche, M. Girod, J. Libert, H. Goutte, S. Hilaire, S. Péru, N. Pillet, and G. F. Bertsch, Phys. Rev. C 81, 014303 (2010).

[36] J. Skalski, S. Mizutori, and W. Nazarewicz, Nucl. Phys. A617, 282 (1997).

[37] F. R. Xu, P. M. Walker, and R. Wyss, Phys. Rev. C 65, 021303 (2002).

[38] Y. Shi, P. M. Walker, and F. R. Xu, Phys. Rev. C 85, 027307 (2012).

[39] A. Petrovici, K. W. Schmid, and A. Faessler, J. Phys. Conf. Ser. 312, 092051 (2011).

[40] T. Togashi, Y. Tsunoda, T. Otsuka, and N. Shimizu, Phys. Rev. Lett. 117, 172502 (2016).

[41] I. Dillmann, K.-L. Kratz, A. Wöhr, O. Arndt, B. A. Brown, P. Hoff, M. Hjorth-Jensen, U. Köster, A. N. Ostrowski, 
B. Pfeiffer, D. Seweryniak, J. Shergur, and W. B. Walters, Phys. Rev. Lett. 91, 162503 (2003).

[42] A. Jungclaus, L. Cáceres, M. Górska, M. Pfützner et al., Phys. Rev. Lett. 99, 132501 (2007).

[43] D. Atanasov et al., Phys. Rev. Lett. 115, 232501 (2015).

[44] F. Browne et al., Phys. Lett. B 750, 448 (2015).

[45] T. Sumikama, K. Yoshinaga, H. Watanabe, S. Nishimura, Y. Miyashita, K. Yamaguchi, K. Sugimoto, J. Chiba, Z. Li et al., Phys. Rev. Lett. 106, 202501 (2011).

[46] T. Kubo et al., Prog. Theor. Exp. Phys. (2012) 03 C003.

[47] N. Fukuda, T. Kubo, T. Ohnishi, N. Inabe, H. Takeda, D. Kameda, and H. Suzuki, Nucl. Instrum. Methods Phys. Res., Sect. B 317, 323 (2013).

[48] S. Takeuchi, T. Motobayashi, Y. Togano, M. Matsushita, N. Aoi, K. Demichi, H. Hasegawa, and H. Murakami, Nucl. Instrum. Methods Phys. Res., Sect. A 763, 596 (2014).

[49] S. Agostinelli et al., Nucl. Instrum. Methods Phys. Res., Sect. A 506, 250 (2003).

[50] P. Doornenbal, Prog. Theor. Exp. Phys. 2012, 3C004 (2012).

[51] A. Obertelli et al., Eur. Phys. J. A 50, 8 (2014).

[52] C. Santamaria et al., Phys. Rev. Lett. 115, 192501 (2015).

[53] NNDC database, Nucl. Instrum. Methods Phys. Res., Sect. A 213 (2011), www.nndc.bnl.gov.

[54] M. Borrajo, T. R. Rodríguez, and J. Egido, Phys. Lett. B 746, 341 (2015).
[55] B. Bally, B. Avez, M. Bender, and P.-H. Heenen, Phys. Rev. Lett. 113, 162501 (2014).

[56] L. Wilets and M. Jean, Phys. Rev. 102, 788 (1956).

[57] See Supplemental Material at http://link.aps.org/ supplemental/10.1103/PhysRevLett.118.032501, which includes Refs. [58-61], for further details of the calculations.

[58] T. R. Rodríguez and J. L. Egido, Phys. Rev. C 81, 064323 (2010).

[59] J. Libert, M. Girod, and J.-P. Delaroche, Phys. Rev. C 60, 054301 (1999).

[60] K. Kumar and M. Baranger, Nucl. Phys. A92, 608 (1967).

[61] W. Ryssens, P.-H. Heenen, and M. Bender, Phys. Rev. C 92 , 064318 (2015).

[62] J. Dechargé and D. Gogny, Phys. Rev. C 21, 1568 (1980).

[63] J. Berger, M. Girod, and D. Gogny, Comput. Phys. Commun. 63, 365 (1991).

[64] M. Bender and P.-H. Heenen, Phys. Rev. C 78, 024309 (2008).

[65] J. Sadoudi, M. Bender, K. Bennaceur, D. Davesne, R. Jodon, and T. Duguet, Phys. Scr. T154, 014013 (2013).

[66] T. Otsuka and Y. Tsunoda, J. Phys. G 43, 024009 (2016).

[67] A. Gade, R. Janssens, D. Weisshaar, B. Brown, E. Lunderberg et al., Phys. Rev. Lett. 112, 112503 (2014).

[68] L. Gaudefroy, A. Obertelli, S. Péru, N. Pillet, S. Hilaire, J.-P. Delaroche, M. Girod, and J. Libert, Phys. Rev. C 80, 064313 (2009).

[69] G. Hagen, P. Hagen, H.-W. Hammer, and L. Platter, Phys. Rev. Lett. 111, 132501 (2013). 